\title{
Extracting Thin-film Optical Parameters from Spectrophotometric Data by Evolutionary Optimization
}

\section{Rajdeep Dutta}

Agency for Science, Technology and Research (A*STAR)

Siyu Isaac Parker Tian

National University of Singapore

Zhe Liu

Massachusetts Institute of Technology

Selvaraj Venkataraj

National University of Singapore

Yuanhang Cheng

National University of Singapore

\section{Daniil Bash}

Agency for Science, Technology and Research (A*STAR)

Tonio Buonassisi

Massachusetts Institute of Technology

Senthilnath Jayavelu ( $\boldsymbol{\sim}$ J_Senthilnath@i2r.a-star.edu.sg )

Agency for Science, Technology and Research (A*STAR)

\section{Research Article}

Keywords: optical parameters, evolutionary optimization, thin-film

Posted Date: November 18th, 2021

DOI: https://doi.org/10.21203/rs.3.rs-1045573/v1

License: (c) (1) This work is licensed under a Creative Commons Attribution 4.0 International License. Read Full License 


\title{
Extracting Thin-film Optical Parameters from Spectrophotometric Data by Evolutionary Optimization
}

\author{
Rajdeep Dutta ${ }^{1,+, *}$, Siyu Isaac Parker Tian, ${ }^{2,+}$, Zhe Liu ${ }^{3}$, Selvaraj Venkataraj ${ }^{4}$, Yuanhang \\ Cheng $^{4}$, Daniil Bash ${ }^{5}$, Tonio Buonassisi ${ }^{3, *}$, and Senthilnath Jayavelu ${ }^{1,6, *}$
}

\author{
${ }^{1}$ Institute for Infocomm Research $\left(I^{2} R\right)$, Agency for Science, Technology and Research (A*STAR), Singapore. \\ ${ }^{2}$ National University of Singapore, Singapore. \\ ${ }^{3}$ Massachusetts Institute of Technology, Cambridge, USA. \\ ${ }^{4}$ Solar Energy Research Institute of Singapore (SERIS), National University of Singapore, Singapore. \\ ${ }^{5}$ Institute of Materials Research and Engineering, Agency for Science, Technology and Research (A*STAR), \\ Singapore. \\ ${ }^{6}$ Artificial Intelligence, Analytics And Informatics $\left(\mathrm{Al}^{3}\right), \mathrm{A}^{*} \mathrm{STAR}$, Singapore. \\ *correspondence to Rajdeep_Dutta@i2r.a-star.edu.sg, buonassi@mit.edu, J_Senthilnath@i2r.a-star.edu.sg \\ +these authors contributed equally to this work
}

\begin{abstract}
Extracting optical parameters from spectrophotometric measurements is a challenging task. In a photometric setup, an unknown thin-film is subjected to an incident light beam for a range of admissible wavelengths, which outputs reflectance and transmittance spectra. The current work attempts to solve an inverse problem of extracting thin-film thickness and complex refractive index from reflectance and transmittance spectra for an incident angle of light. The film thickness is a scalar quantity, and the complex refractive index is composed of real and imaginary parts as functions of wavelengths. We leverage evolutionary optimization techniques to solve the underlying inverse problem, which determines the desired parameters associated with two optical dispersion models: ensemble of Tauc-Lorentz (TL) and ensemble of Gaussian oscillators, such that the generated spectra accurately fit the input data. The optimal parameters involved in the adopted models are determined using efficient evolutionary algorithms (EAs). Numerical results validate the effectiveness of the proposed approach in estimating the optical parameters of interest.
\end{abstract}

\section{Introduction}

The determination of a thin-film's thickness and complex refractive index over a broad spectral range is carried out using either ellipsometric or spectrophotometric analysis ${ }^{1,2}$. Ellipsometry measures how the polarization of a light beam changes on reflection from a surface. Spectrophotometry measures reflectance and/or transmittance of light through thin-films and substrates as function(s) of wavelength. Reflectance and/or transmittance spectral information are further fed to a numerical solver for extracting thickness and refractive index ${ }^{3,4}$. Thickness influences micro to macro-scale physics, including from electron mobility in thin-film transistors ${ }^{5}$ to operating characteristics of thin-film gas sensors ${ }^{6}$. Refractive index based detectors play a significant role in characterizing the changes of several chemical and biological measurands, like gas concentration and water $\mathrm{pH}$ values ${ }^{7}$. Complex refractive index profiles are crucial in designing appropriate polymers to produce effective lenses and ultraviolet (UV)-absorbing coatings ${ }^{8}$. Thickness variations by adjusting the catalyst concentration or changing the heat-treatment process can be leveraged to shift the reflection band of a film from narrow to broad wavelength region, which is beneficial in fabricating dielectric reflectors for solar cells and band pass filters for optical instruments ${ }^{9}$. For compounded materials, an accurate estimation of a film's optical parameters is required to optimize the deposition process.

Computationally, it is quite nontrivial to achieve accurate estimates of all the optical parameters together ${ }^{10}$. A method that gives the most accurate solution for thickness, does not usually give the most accurate solution for complex refractive index ${ }^{11,12}$. Moreover, different methods perform well in different spectral ranges ${ }^{12,13}$. Two inherent difficulties in an inverse photometric problem are: (a) missing information induced by experimental uncertainty or film imperfections, and (b) multiple solutions due to the fact that the measured intensities might be compatible with other plausible combinations of optical constants ${ }^{14}$. To dissolve the ambiguity in parameter extraction, previous researchers ${ }^{12}$ exploited reflectance and transmittance spectral measurements at two distinct incident angles of light. Thus, there emerges a need of developing efficient methods for the 
precise determination of not only thickness but also complex refractive index. Several commercial packages of thin-film optical software, such as TFCalc, Filmwizard, Optilayer, and Essential McLeod, provide advanced modelling for optical dispersion laws and use fitting methods to determine the thickness and complex refractive index ${ }^{11}$. However, these packages cannot always find satisfactory solutions, especially when the initial candidate is far away from the desired solution in case of a strongly absorbing material like silicon in the visible range ${ }^{11}$. A recent development, RefDex ${ }^{4,15}$ provided an interactive fitting procedure to extract the complex refractive index of a film with known thickness from reflectance and transmittance data. This inverse problem is not unique and there exist multiple solutions that minimize the same loss function ${ }^{4}$, which mandates imposing physical constraints to determine meaningful solutions. The major concern is that the above-mentioned software-packages do not offer much flexibility on the choice of parameters, such as the number of oscillators and the bounds on model coefficients, involved in their respective optimization procedures.

Refractive index and extinction coefficient profiles can be substantially different for various materials ${ }^{16}$. For instance, consider some of the metal oxides; the complex refractive indices of the iron oxides, Hematite and Magnetite, exhibit more peaks and valleys than that of the copper oxides ${ }^{17}$. Due to this fact, a supervised learning approach confronts challenges in predicting refractive indices for a broad range of materials ${ }^{18}$. A supervised method that is trained with inorganic material data, might not give satisfactory prediction performance in the case of organic materials ${ }^{19}$. Thus, there arises a need for transfer learning in combination with supervised learning. On the contrary, an evolutionary optimization approach has the potential to bypass the requirement of intense training with labelled data. That is why, in the present work, we pose the underlying inverse problem as an optimization problem and solve it using evolutionary algorithms.

Earlier studies explored optimization-based fitting procedures to extract optical parameters from spectrophotometric data. Woollam et al. ${ }^{20,21}$ proposed a tool that allowed sequential addition of various optical models and simultaneously minimized fitting errors to determine refractive index and thickness. A local optimizer, Levenberg-Marquardt (LM) algorithm ${ }^{22}$, was utilized in their approach, which gradually improved the solution accuracy. The optimizer starts from an initial guess and moves it to a feasible local minimum until another model addition becomes necessary for further reduction in the fitting error. Swarm-based evolutionary algorithms (EAs) are robust and good at finding the global optimum even for high-dimensional problems $^{23,24}$, whereas single point based gradient descent variant algorithms face challenges due to local entrapment and require good initial guesses to reach the global optimum ${ }^{22,25}$. EAs have also been exploited to determine the optical parameters of interest ${ }^{26}$. Genetic algorithm (GA) and simulated annealing (SA) have been exploited in ellipsometric evaluations, where the traditional gradient-based LM method faces difficulty in tackling the related hilly error surfaces ${ }^{27}$. Gao et al. ${ }^{11}$ applied GA and SA on an ensemble of Tauc-Lorentz oscillators to extract the refractive index real and imaginary parts of a film with known thickness from reflectance and transmittance spectra. However, their approach required a large population to determine the desired inverse solutions. In EAs, usually a small population size saves the cost of computing functions whereas a large population size enhances the search space exploration and helps in avoiding local optima at the cost of computational overhead. In recent years, covariance matrix adaptation evolution strategy (CMAES) has proved to be reliable in solving deterministic or stochastic global optimization problems even with a small population size due to the attributes like step-size adaptation, noise effect reduction, and invariance under coordinate systems ${ }^{28}$. The present work aims at determining a thin-film's complex refractive index and thickness with the help of EAs, such as GA and CMAES.

In this paper, we adopt EAs to solve the inverse problem of extracting a thin-film's optical parameters from spectrophotometric data of reflectance and transmittance spectra at just one incident angle of light. Further, the current solution approach can be applied to a variety of materials. The following first describes the related optical models along with the proposed optimization methodology to solve the underlying inverse problem, and then presents the numerical results obtained by the same. Finally, we discuss the major traits of the achieved inverse solutions and conclude the current work.

\section{Physics to Mathematics}

In our spectrophotometric analysis, the optical parameters to be extracted are: thickness $(d)$, refractive index $(n)$, and extinction coefficient $(k)$, and the measured data are: reflectance $(R)$ and transmittance $(T)$ spectra. A thin-film is situated in between air and substrate mediums in a photometric set-up, as shown by Figure 1 . The film material has real and imaginary constituents in its complex refractive index profile. The real part (or refractive index) describes the propagation velocity of the incident light within the material, and the imaginary part (or extinction coefficient) concerns about how much of it gets absorbed in the medium. This study aims to solve the inverse problem of determining $\{d, n, k\}$ from $\{R, T\}$. The following describes the use of optical oscillator models to emulate complex refractive index $(n+i k)$ profiles required in the solution procedure.

\section{Forward Calculations via Oscillator Models}

The complex dielectric function, $\varepsilon(\omega)=\varepsilon_{1}(\omega)+i \varepsilon_{2}(\omega)$, is analytic in the upper half of the complex $\omega$ plane ${ }^{16,29}$, where $\omega=\frac{c}{\lambda}$ denotes the frequency of the incident light with $\lambda$ being its wavelength and $c$ being the speed of light in air. The associated photon energy is represented by $E=h \omega ; h$ is Planck's constant. The analytic behavior of $\varepsilon(\omega)$ stems from the 


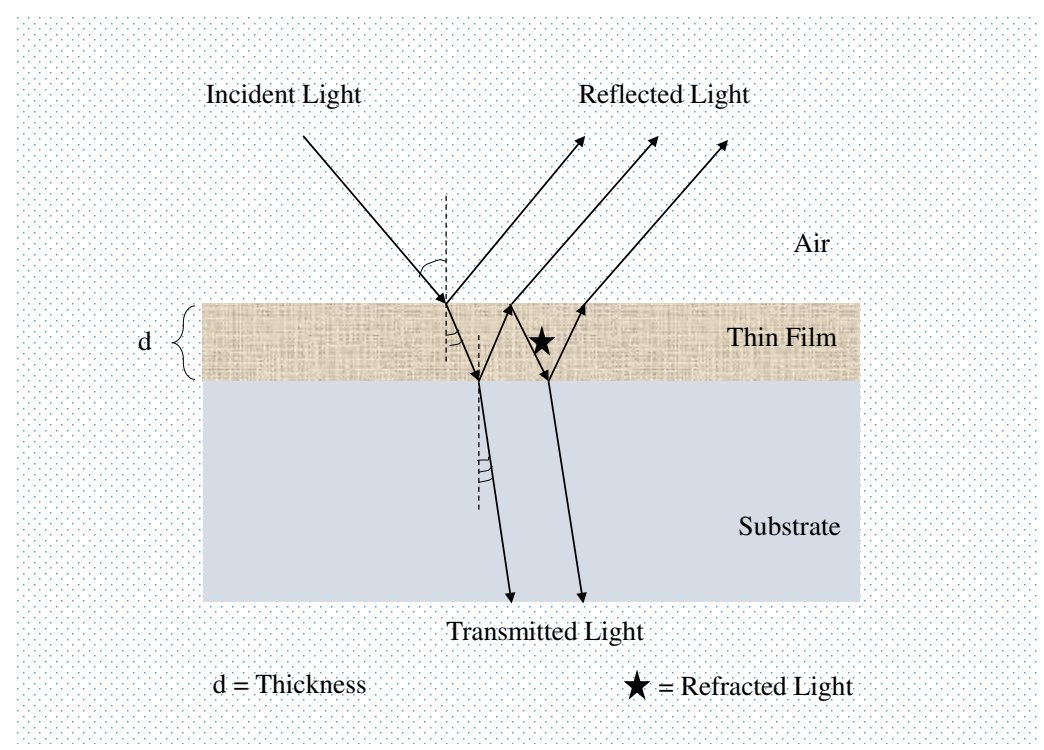

Figure 1. Placement of a thin-film in the photometric experiment.

principle of causality ${ }^{16,30}$. As a consequence, the imaginary and real parts of it are interconnected by the Kramers-Kronig relation $^{30}$. Using this relation ${ }^{29}$, one can determine $\varepsilon_{1}$ from $\varepsilon_{2}$ as follows.

$$
\varepsilon_{1}(\omega)=\varepsilon_{1}(\infty)+\frac{2}{\pi} P \int_{0}^{+\infty} \frac{\Omega \varepsilon_{2}(\Omega)}{\Omega^{2}-\omega^{2}} d \Omega,
$$

where $P$ denotes Cauchy's principle value integra ${ }^{16}$. The complex dielectric function and complex refractive index are related by: $\varepsilon_{1}+i \varepsilon_{2}=(n+i k)^{2}$, which leads to

$$
n^{2}(\omega)-k^{2}(\omega)=\varepsilon_{1}(\omega) \& 2 n(\omega) * k(\omega)=\varepsilon_{2}(\omega) .
$$

By substituting Equation (2) into Equation (1), the dispersion relation ${ }^{16,31}$ between the real $(n)$ and imaginary $(k)$ parts of a complex refractive index can be unravelled as

$$
n(\omega)=n(\infty)+\frac{2}{\pi} P \int_{0}^{+\infty} \frac{k(\Omega)}{\Omega^{2}-\omega^{2}} d \Omega .
$$

To accurately evaluate $n(\omega)$ from $k(\omega)$, the above integration (3) requires to be solved for all frequencies, ranging from zero to infinity ${ }^{16,30}$. However, from an experimental perspective, it is only feasible to address a finite range of frequencies. A numerical integration with such a limited spectral range gives erroneous results ${ }^{31}$. Instead, if the functional form of $k(\omega)$ is known for all frequencies, then the functional form of $n(\omega)$ can be determined elegantly ${ }^{16}$. Therefore, oscillator models are utilized to generate efficient continuous function approximations. In this research, we take Tauc-Lorentz and Gaussian oscillator models into consideration, as described briefly in the following.

Ensemble of Tauc-Lorentz Oscillators: Consider a material with the complex dielectric function: $\varepsilon=\varepsilon_{1}+i \varepsilon_{2}$. According to an ensemble of $N$ Tauc-Lorentz (TL) oscillators ${ }^{11}$, the imaginary part of the complex dielectric function can be expressed as

$$
\varepsilon_{2}(\omega)=\left\{\begin{array}{l}
\sum_{i=1}^{N} \frac{A_{i} \omega_{0_{i}} C_{i}\left(\omega-\omega_{g}\right)^{2}}{\left(\omega^{2}-\omega_{g}^{2}\right)^{2}+C_{i}^{2} \omega^{2}} \frac{1}{\omega} \quad \text { for } \omega>\omega_{g} \\
0 \text { for } \omega \leq \omega_{g},
\end{array}\right.
$$

where $\omega_{0_{i}}, \omega_{g}, C_{i}, A_{i}$ represent the peak transition frequency, the band gap frequency, the broadening parameter, and the factor involving optical transition matrix elements for the $i$ 'th TL oscillator, respectively. To calculate $\varepsilon_{1}$ from $\varepsilon_{2}$, let us now recall the Kramers-Kronig relation (1). In practice, the lower limit of the integral in Equation (1) is chosen as $\omega_{g}$ instead of 0 because the Tauc-Lorentz model requires $\varepsilon_{2}$ to be zero for photon energies below the band gap ${ }^{29}$. Note that $\varepsilon_{1}(\infty)>1$ is a high frequency dielectric constant to prevent $\varepsilon_{1} \rightarrow 0$ when $\omega<\omega_{g}$. 
The formulation of these optical functions was first proposed by Forouhi and Bloomer for amorphous semiconductors and insulators ${ }^{16}$, and later, extended for crystalline semiconductors and metals ${ }^{30}$. According to the seminal work by Jellison and Modine $^{29}, \varepsilon_{1}(\omega)$ can be derived from Equation (1) by exploiting the continuous approximation (4) of $\varepsilon_{2}(\omega)$. These $\varepsilon_{1}$ and $\varepsilon_{2}$ are then used to solve Equation (2) for all $\omega$, so that $n$ and $k$ can be deduced as a function of ( $3 N+3$ ) parameters, where $3 N$ parameters come from $\left(\omega_{0_{i}}, C_{i}, A_{i}\right)$ for $i \in[1, N]$ and the rest three parameters are $d, \omega_{g}$, and $\varepsilon_{1}(\infty)$. The number of decision variables involved in optimizing a TL ensemble model are $(3 N+3)$.

Ensemble of Gaussian Oscillators: The imaginary r-index profile according to a Gaussian oscillator model, is given by

$$
k(\omega)=\sum_{i=1}^{N} A_{i} \exp \frac{-\left(w-\mu_{i}\right)^{2}}{2 \sigma_{i}^{2}}
$$

where, $N$ is the number of Gaussian functions to be mixed; the parameters associated with the $i$ 'th Gaussian function are $A_{i}, \mu_{i}, \sigma_{i}$ denoting its amplitude, mean and variance, respectively ${ }^{32}$. Next, $n(\omega)$ is determined by applying the Kramers-Kronig integration (3) to the continuous approximation (5) of $k(\omega)$, which takes shape as

$$
n(\omega)=n(\infty)+\frac{2}{\sqrt{\pi}} \sum_{i=1}^{N} A_{i} \exp ^{-W_{i}^{2}} \int_{0}^{-W_{i}} \exp ^{x^{2}} d x, W_{i}=\frac{\left(w-\mu_{i}\right)}{\sqrt{2} \sigma_{i}},
$$

where $n(\infty)=1$ refers to $k(\infty)=0$ and $n(\infty)>1$ refers to $k(\infty) \neq 0^{30,32}$. Here, Gaussian distributions are utilized to retrieve $n$ and $k$ directly instead of deriving from $\varepsilon_{1}$ and $\varepsilon_{2}$. An ensemble of Gaussian oscillators (GO) composed of $N$ Gaussian distributions, deals with $3 N$ parameters as $A_{i}, \mu_{i}, \sigma_{i}$ for $i \in[1, N]$ and two more parameters as $\bar{n}$ and $d$. Thus, the total number of decision variables involved in optimizing a GO ensemble model are $(3 N+2)$.

Note that the above optical characteristics $n(\omega)$ and $k(\omega)$ are expressed as $n(\lambda)$ and $k(\lambda)$, respectively, during the numerical implementation. The order of $(n, k)$ sequences gets reversed, ascending to descending, when the independent variable $\omega$ changes to $\lambda$ since $\omega=\frac{c}{\lambda}$. The forward calculation of $\left\{R_{\text {calc }}(\lambda), T_{\text {calc }}(\lambda)\right\}$ for a tuple $\{d, k(\lambda), n(\lambda)\}$ is carried out by the transfer-matrix method ${ }^{13}$. The transfer-matrix method is used to calculate the forward and backward propagating electric fields in smooth homogeneous films, and it relies on the superposition of electric fields. The overall transfer matrix is obtained by multiplying a matrix that quantifies the change in field due to light waves propagating through an interface (air-to-film) with another matrix that quantifies the change in field due to the same waves propagating within a layer (film).

\section{Inverse Problem Formulation}

We now explain how the underlying inverse problem is posed as an optimization problem. An overview of the associated forward and inverse processes is depicted in Figure 2.

Optimization Problem: The optimization problem is defined as

$$
\min _{d, k(\lambda), n(\lambda)} L=\sum_{\lambda=\lambda_{l b}}^{\lambda_{u b}}\left\{R_{\text {meas }}(\lambda)-R_{\text {calc }}(\lambda)\right\}^{2}+\left\{T_{\text {meas }}(\lambda)-T_{\text {calc }}(\lambda)\right\}^{2}
$$

In Equation (7), $L$ is the total loss containing differences in the experimentally measured reflectance and transmittance $\left\{R_{\text {meas }}, T_{\text {meas }}\right\}$ from their theoretically calculated values $\left\{R_{\text {calc }}, T_{\text {calc }}\right\}$, for all wavelengths. The admissible range of the wavelength, i.e. $\left\{\lambda_{l b}, \lambda_{u b}\right\}$, depends on the experimental infrastructure. The decision variables associated with the optimization problem, are: thickness $d$, real r-index profile $n(\lambda)$, and imaginary r-index profile $k(\lambda)$. Thickness candidates are directly passed to the solver, whereas $n$ and $k$ candidates are passed in terms of the oscillator model parameters. The evaluation of $\left\{R_{\text {calc }}(\lambda), T_{\text {calc }}(\lambda)\right\}$ for a candidate solution $\{d, n(\lambda), k(\lambda)\}$ has been discussed in the preceding section.

In order to make the optimization approach computationally efficient, we generate candidate solutions within a search space restricted by an intrinsic correlation ${ }^{16,31}$ between the optical parameters of interest. The thickness candidates are chosen from a reasonable range of scalars. The imaginary and real refractive index candidate profiles are provided by the adopted oscillator models. A formulation over a range of wavelengths is computationally more tractable than a formulation at distinct wavelengths. In a discrete approach, the search space increases with the number of wavelengths and it is cumbersome to determine a physically meaningful refractive index (and/or extinction coefficient) profile out of every solution point at each wavelength, while satisfying the associated constraints. Whereas in our optimization approach, the parameter search space gets restricted by the Kramers-Kronig relation during candidate generation.

\section{Data Curation}

The proposed methodology is implemented on a diverse data set containing two kinds of thin-film materials: (A) metal oxide thin-films, and (B) perovskite thin-films. 


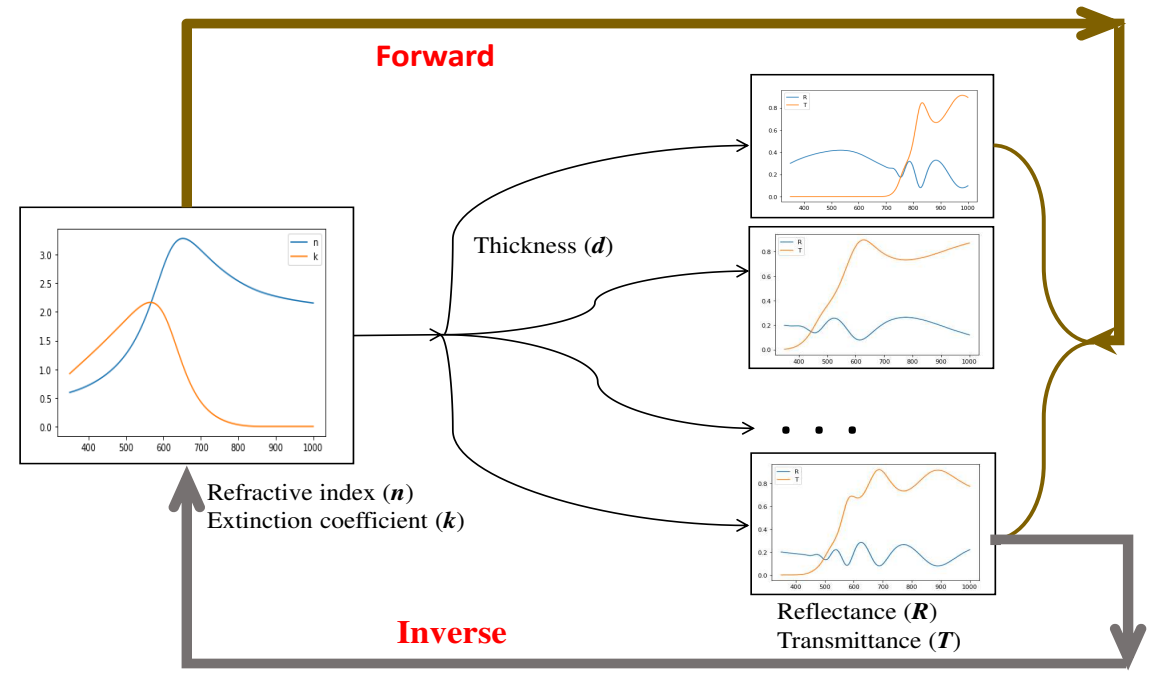

Figure 2. A flow diagram of the involved forward and inverse processes. (a) Forward: For a specific material with certain $n(\lambda)$ and $k(\lambda)$ profiles, different $d$ values produce different spectra $\{R(\lambda), T(\lambda)\}$; (b) Inverse: From the measured data $\{R(\lambda), T(\lambda)\}$, the reverse engineering task is to find a solution: $\{d, n(\lambda), k(\lambda)\}$.

We obtain the synthetic data for two classes of the datasets, the Tauc-Lorentz (TL) dataset whose refractive index follows a single Tauc-Lorentz oscillator (commonly used for metal oxide materials), and the perovskite data set whose refractive index is from perovskite materials ${ }^{33}$. There are two steps involved in preparing the synthetic data: 1) obtain refractive indices $n$ and $k$ either from the simulation (for TL dataset) or from the literature ${ }^{33}$ (for perovskite dataset); 2) obtain the reflectance $R$, and transmittance $T$ by using the transfer-matrix method with the $(n, k)$ profiles obtained in step (1) and user-specified $d$ values, where the wavelength range selected in the UV-Vis-NIR is $350-1000 \mathrm{~nm}$. For TL dataset, Step (1): 1,116 $(n, k)$ spectra were simulated with a python implementation of a one-oscillator Tauc-Lorentz model. Step $(2): 15,640(R, T)$ spectra were simulated with a python implementation of the transfer-matrix method by assigning 10 random thickness values $d$ in the range of $20-2000 \mathrm{~nm}$ throughout the dataset. Unlike the TL dataset, the perovskite dataset only requires one-step simulation, i.e., the simulation of $(R, T)$ spectra using the transfer-matrix method, while a variety of $18(n, k)$ profiles are picked from the literature ${ }^{33}$.

To generate an experimental data, the $\mathrm{MAPbI}_{3}$ perovskite film is deposited on glass substrate with two process variables affecting thickness, which are the concentration $(M=1.25)$ of the perovskite precursor solution $\left(\mathrm{PbI}_{2}\right.$ and $\mathrm{MAI}$ with molar ratio of 1:1) and the spin coating speed (r.p.m.=3000). We conduct two characterizations: (1) spectrophotometry (UV-Vis), with an Agilent Cary 7000 UV-Vis-NIR Spectrophotometer ${ }^{34}$, to obtain the optical reflection and transmission (2) profilometry, with a KLA Tencor P-16 + Plus Stylus Profiler, to obtain thickness of deposited films.

\section{Numerical Results}

Implementation Aspects: During the implementation for extracting thin-film optical characteristics, wavelength $(\lambda)$ is considered as the independent variable. Reflectance and transmittance spectra are measured for a wavelength range of 350 to $1000 \mathrm{~nm}$. The inverse problem of determining $d, n(\lambda), k(\lambda)$ from $R, T$ is not unique. So, there is a risk that an optimization algorithm unravels solutions that do not make sense physically. To mitigate this issue, we pose bounds on the decision variables involved in TLO and GO models such that negative $n$ and $k$ are always discouraged during the optimization process. The decision variables associated with the TLO ensemble model, i.e. $A_{i}, \omega_{0_{i}}, C_{i}, \omega_{g}, \varepsilon_{\infty}, d$, are selected from a bounded space: $\{0,100\},\left\{0, \frac{10}{h}\right\},\{0,10\},\left\{0, \frac{5}{h}\right\},\{0,2\},\{20,2000\}$. In TL ensemble model, 2,3 and 4,5 oscillator components are chosen for type A and B materials, which gives rise to 9,12 and 15, 18 variables to be optimized, respectively. For each TLO oscillator, two parameter conditions: $\left(\omega_{0_{i}}>\omega_{g}\right)$ and $\left(h \omega_{0_{i}}>C_{i} / \sqrt{2}\right)$ are maintained during optimization via penalizing the objective function value. The decision variables associated with the GO model, i.e. $A_{i}, \mu_{i}, \sigma_{i}, \bar{n}, d$, are selected from a bounded space: 
$\{0,5\},\{3,9\},\{0.2,1.1\},\{0,5\},\{20,2000\}$. In GO ensemble model, 2,3 and 4,5 oscillator components are chosen for type A and B materials, giving rise to 8,11 and 14,17 variables to be optimized, respectively.

Choice of Algorithms: We apply two EAs, namely Genetic Algorithm (GA) and Covariance Matrix Adaptation Evolution Strategy (CMAES), for optimizing the parameters associated with the adopted optical dispersion models: TLO ensemble and GO ensemble. To validate the performance of CMAES and GA on TLO as well as GO ensembles, 20 samples are drawn randomly from the synthetic data set. For each sample, three runs are considered with a maximum of 200 iterations and the best result is stored. Table 1 presents a performance comparison between the employed local and global optimization algorithms, i.e. LM vs. GA vs. CMAES. The average loss is drastically higher in case of the local optimization algorithm than the global optimization algorithms because the former drives only a single point that may easily get trapped by local optima whereas the latter drives a population of candidates to efficiently explore the search space while avoiding local traps. Therefore, the performance of LM very much depends on the initial condition; a better starting point leads to a lower loss value. On the contrary, the performance of EAs like GA or CMAES is less sensitive to the initial population. Table 1 shows that the average loss achieved by CMAES is the lowest. This study bolsters the use of CMAES over GA in the underlying inverse problem.

\begin{tabular}{|c|c|c|c|}
\hline Model Algorithm & LM & GA & CMAES \\
\hline \hline TLO & 61.6164 & 3.0407 & 1.3828 \\
\hline GO & - & 2.5997 & 2.4713 \\
\hline
\end{tabular}

Table 1. Performance evaluation of different optimization algorithms.

Performance Evaluation: CMAES is applied onto 100 samples of type A and 100 samples of type B thin-films, randomly drawn from the synthetic data set. For each sample, five runs are considered with a maximum of 1500 iterations and the best result is stored. We cut the run if CMAES reaches a loss value below 0.05 (stopping criteria). Note that a loss value of 0.05 in Equation (7), refers to a mean square error of $0.05 / 651=7.68 E-5$ for $(1000-350)+1=651$ wavelengths in the given range. A successful occasion is counted if the thickness estimation error is below $10 \%$ along with a minimum loss of $\leq 0.17$ (saving criteria). Table 2 reports all the associated metrics, such as: $E E_{d}$ denotes the $\mathrm{R}^{2}$-score between the original thickness and estimated thickness values, $m E E_{n}$ denotes the median of the sequence of $\mathrm{R}^{2}$-scores between the original and estimated $n(\lambda)$ arrays, $m E E_{k}$ denotes the median of the sequence of $\mathrm{R}^{2}$-scores between the original and estimated $k(\lambda)$ arrays, $m E E_{R}$ denotes the mean of the sequence of $\mathrm{R}^{2}$-scores between the original and estimated $R(\lambda)$ arrays, $m E E_{T}$ denotes the mean of the sequence of $\mathrm{R}^{2}$-scores between the original and estimated $T(\lambda)$ arrays, $S R$ denotes the success rate $=$ number of successful occasions $\left(n_{s s}\right) /$ total occasions $\left(n_{s}\right)$, and $m F E$ is the average number of function evaluations: $\left(1 / n_{s s}\right) * \sum_{i=1}^{n_{s s}} F E_{i}$. In optical parameter estimation, optimizing the TLO ensemble model proves to be more efficient than optimizing the GO ensemble. For successful occasions, optimizing TLO ensemble requires less function evaluations than optimizing GM models.

\begin{tabular}{|c|c||c|c|c|c|c|c|c|}
\hline Thin & \multicolumn{1}{l||}{ Optical } \\
Film & Model & $E E_{d}$ & $m E E_{n}$ & $m E E_{k}$ & $m E E_{R}$ & $m E E_{T}$ & $S R$ & $m F E$ \\
\hline \hline \multirow{4}{*}{ A } & TLO -2 & 0.99502 & 0.90654 & 0.99779 & 0.93573 & 0.98083 & 0.62 & 228.32 \\
& TLO -3 & 0.99500 & 0.94185 & 0.99637 & 0.96052 & 0.98018 & 0.57 & 266.59 \\
\cline { 2 - 9 } & GO - & 0.99884 & -1.41127 & 0.76782 & 0.94592 & 0.87954 & 0.23 & 294.87 \\
& GO -3 & 0.99627 & 0.25544 & 0.95294 & 0.96604 & 0.99185 & 0.27 & 558.15 \\
\hline \hline \multirow{4}{*}{ B } & TLO -4 & 0.99672 & 0.87354 & 0.95219 & 0.98533 & 0.99968 & 0.63 & 755.65 \\
& TLO -5 & 0.99750 & 0.79227 & 0.94078 & 0.98655 & 0.99969 & 0.48 & 747.08 \\
\cline { 2 - 8 } & GO -4 & 0.99996 & 0.30902 & 0.79027 & 0.98416 & 0.99895 & 0.36 & 1161.11 \\
& GO -5 & 0.99997 & 0.44936 & 0.87493 & 0.98771 & 0.99921 & 0.43 & 1321.56 \\
\hline \hline
\end{tabular}

Table 2. Estimation performance of the adopted oscillator ensemble models applied on 100 samples of type A and B films randomly picked from the total synthetic data set.

Moreover, we applied local and global optimization algorithms, i.e. LM, GA and CMAES, on TLO and GO models to extract a perovskite thin-film's (unknown $n, k$ ) optical characteristics from the experimentally measured spectrophotometric data, $(R, T)$. For each method, the respective optimizers are run ten times and the best results are recorded. The maximum number of iterations is set as 1000 , however, we cut the run if the loss function value goes below 0.05 . The corresponding thickness estimates and optimization loss outcomes are listed in Table 3. Figure 6 exhibits the fitting performance of the CMAES algorithm applied on two optical models, along with the extracted complex refractive index profiles. Interestingly, in 


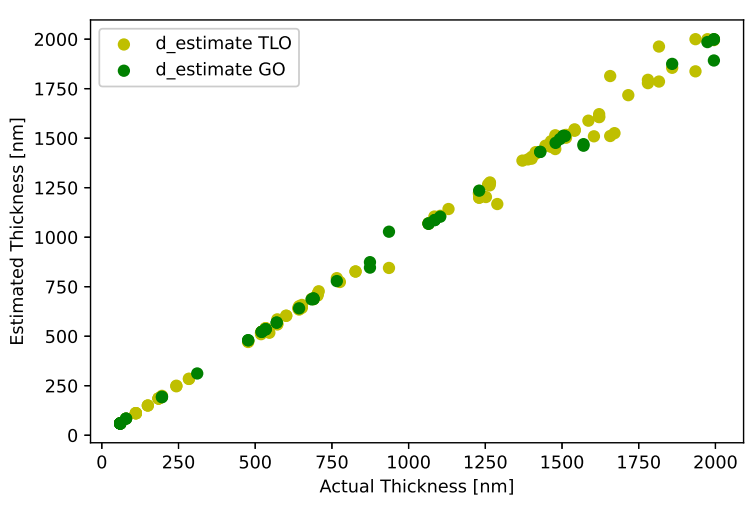

(a)

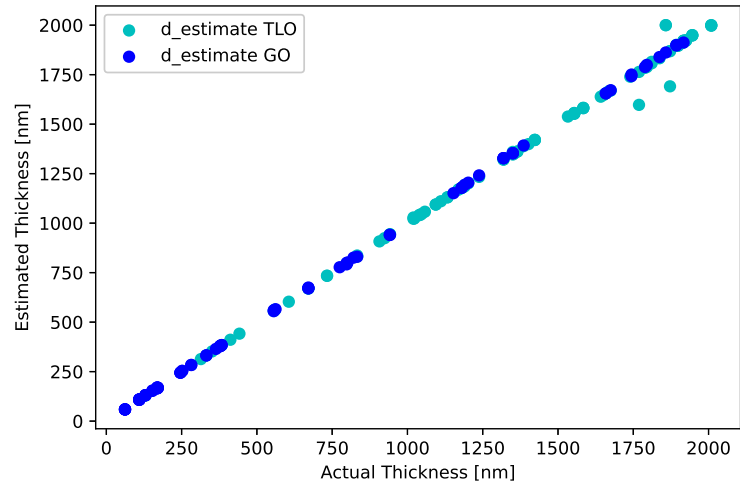

(b)

Figure 3. Visual representation of the actual vs. estimated thickness for the successful occasions reported in Table 2: (a) thickness estimation for type A thin-films, (b) thickness estimation for type B thin-films.

this case, the optimized GO model gives better estimates of $d, n(\lambda), k(\lambda)$ than the optimized TLO model.

\begin{tabular}{|c|c|c|c|c|c|}
\hline Details Methods & TLO+CMAES & GO+CMAES & TLO+LM & $\mathrm{TLO}+\mathrm{GA}$ & $\mathrm{GO}+\mathrm{GA}$ \\
\hline$\overline{\mathrm{d}}$ & 243.71 & $\overline{99.32}$ & $\overline{99.82}$ & 98.07 & $\overline{95.88}$ \\
\hline $\mathrm{L}$ & 2.8513 & 0.0049 & 6.9499 & 0.4035 & 0.2464 \\
\hline $\mathrm{nFE}$ & 9480 & 7332 & 232 & 18000 & 18000 \\
\hline
\end{tabular}

Table 3. Estimation performance of various methods on the experimental data.

Inference from Results: Figure 3 shows all the thickness estimates of various samples for the successful occasions. According to Figure 3(a) and 3(b), the optimized TLO ensemble model finds more number of accurate thickness estimates (within $10 \%$ of the original values) than the optimized GO ensemble model, which is also supported by Table 2 . The performance of the adopted models in estimating refractive index $(n)$ and extinction coefficient $(k)$, can be visualized by the examples shown in Figures 4 and 5. It comes out that capturing $n$ and $k$ profiles simultaneously with thickness estimation is a challenging task. Therefore, $m E E_{n}$ and $m E E_{k}$ in Table 2 are slightly worse even though $E E_{d}$ is quite good. Among Tauc-Lorentz oscillator ensembles, TLO-2 and TLO-4 prove to be efficient and among Gaussian oscillator ensembles, GO-3 and GO-5 are effective. Overall, TLO ensemble optimization serves better than GO ensemble optimization. In Table 2, we mention the medians $m E E_{n}$ and $m E E_{k}$ as the associated $\mathrm{R}^{2}$-score sequences contain outliers that might skew the average of scores. An outlier refers to a solution where the real or imaginary refractive index estimates are inaccurate, although the corresponding thickness estimate is satisfactory. In Figure 4(b), $n$ and $k$ estimates exactly match with the original profiles throughout, however, in Figures 4(d), 5(b) and 5(d), there exist differences between the original and estimated $n, k$ profiles especially in low-wavelength regime, partially including UV and visible spectral ranges. To further inspect this quantitatively, we split the entire wavelength range into two sectors and highlight the estimation performance of the well fitted models as per Table 2.

- TLO-2 for type A films: For a wavelength range of $350-500 \mathrm{~nm}$, the estimation metrics are $m E E_{n}=0.41617, m E E_{k}=$ 0.98999 ; and for a wavelength range of $500-1000 \mathrm{~nm}$, the estimation metrics are $m E E_{n}=0.83078, m E E_{k}=0.99870$.

- TLO-4 for type B films: For a wavelength range of $350-500 \mathrm{~nm}$, the estimation metrics are $m E E_{n}=-0.47954, m E E_{k}=$ 0.72331 ; and for a wavelength range of $500-1000 \mathrm{~nm}$, the estimation metrics are $m E E_{n}=0.97758, m E E_{k}=0.95517$.

The above reported results reveal that the fitting error in $k(\lambda)$ gets amplified into $n(\lambda)$ as it propagates through the KramersKronig integration. In low-wavelength regime, $m E E_{n}$ and $m E E_{k}$ deteriorate seriously for perovskite materials. A possible reason is the presence of intra-band absorption giving rise to $\varepsilon_{2}$ for photon energies below the band gap, which can not be captured well by the Tauc-Lorentz model approximation due to an underlying assumption ${ }^{29}: \varepsilon_{2}(\omega)=0$ for $\omega<\omega_{g}$.

The numerical results justify that the TLO ensemble model performs well for the synthetic data of both type A and B thin-films. The estimation error metrics reported in Table 2 reveal the challenge in achieving a good accuracy of $n(\lambda), k(\lambda)$ 


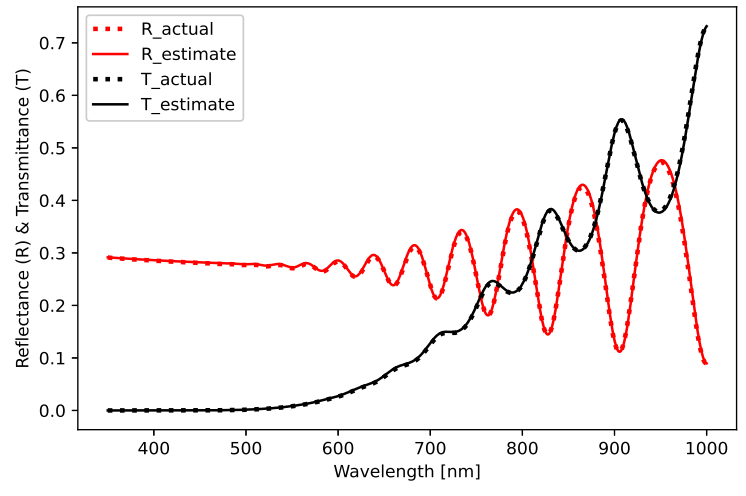

(a)

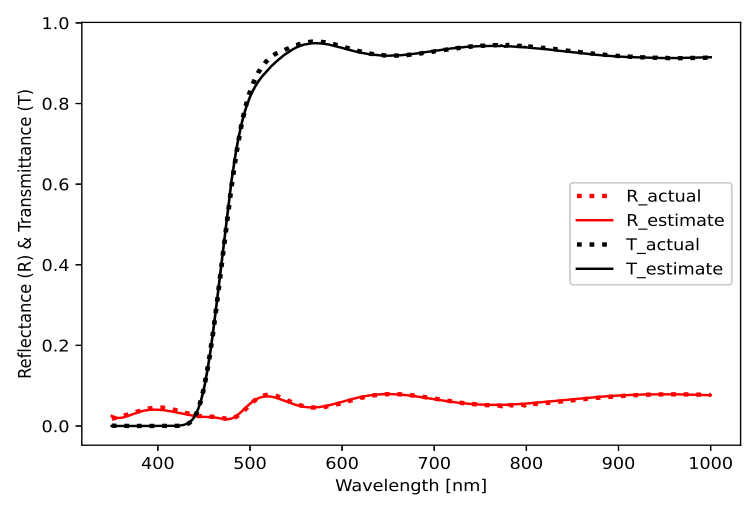

(c)

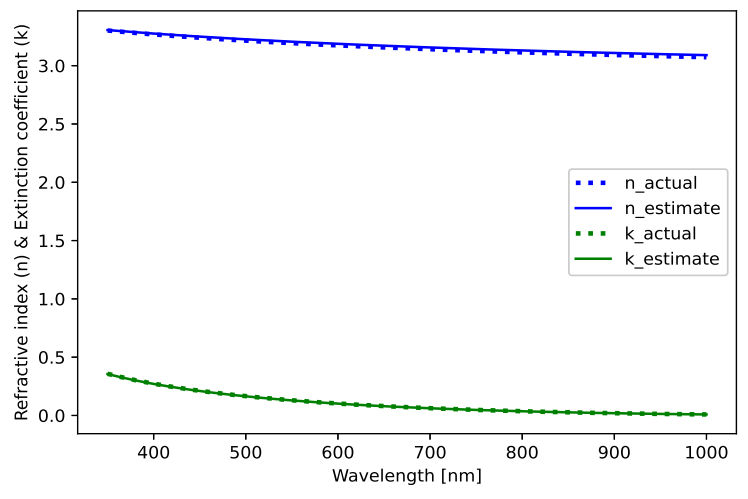

(b)

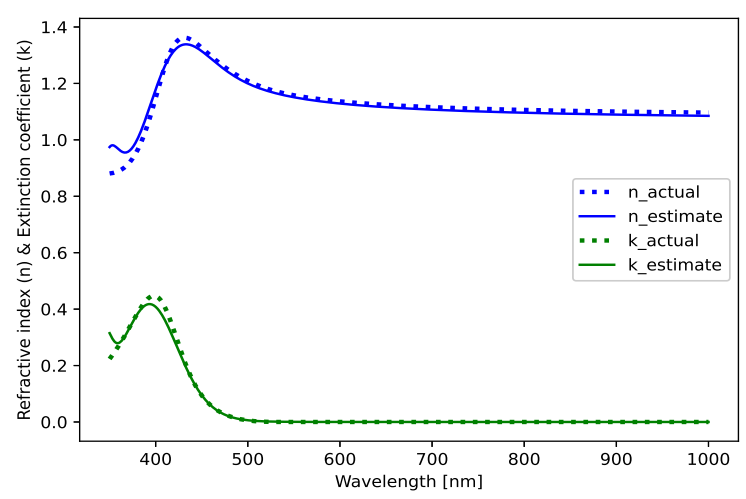

(d)

Figure 4. Inverse solutions obtained from optimized TLO and GO ensemble models applied on spectral data (synthetic) of specimen A: (a) Actual and estimated spectra from optimized TLO ensemble; total estimation loss is: 0.0079, (b) Actual and estimated refractive index and extinction coefficient extracted from optimized GO ensemble; actual and estimated thickness values are: $1464.0,1456.45 \mathrm{~nm}$, (c) Actual and estimated spectra from optimized GO ensemble; total estimation loss is: 0.0272, (d) Actual and estimated refractive index and extinction coefficient extracted from optimized GO ensemble; actual and estimated thickness values are: $874.0,873.91 \mathrm{~nm}$. 


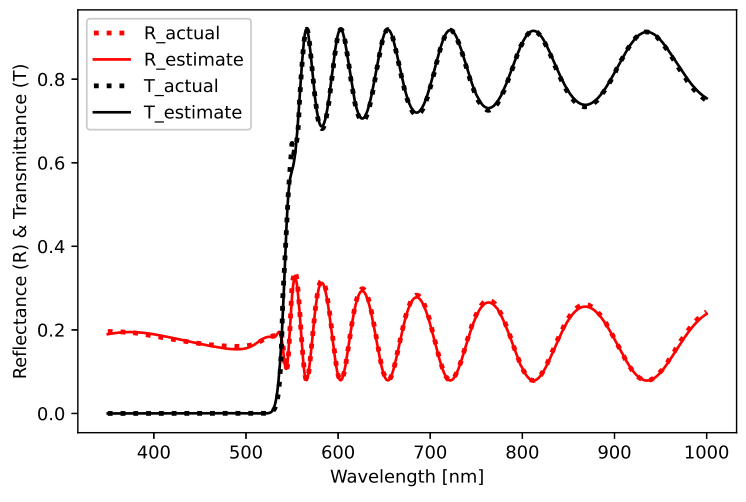

(a)

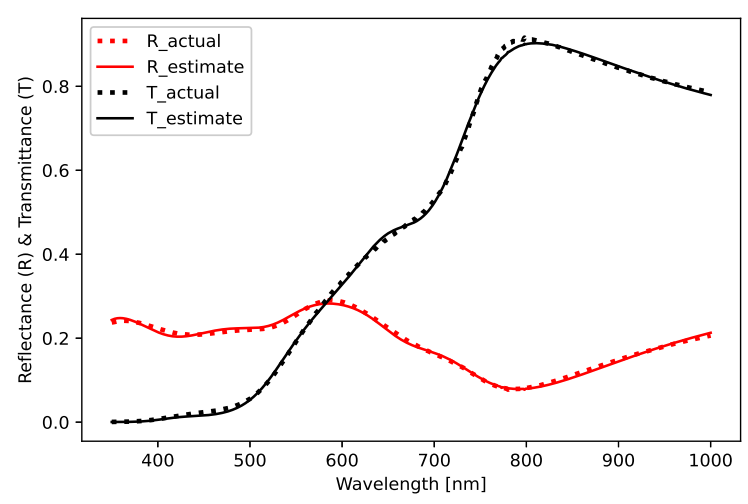

(c)

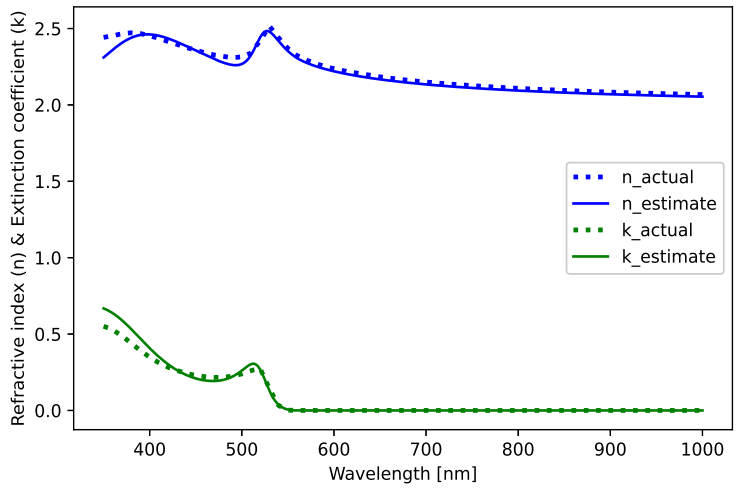

(b)

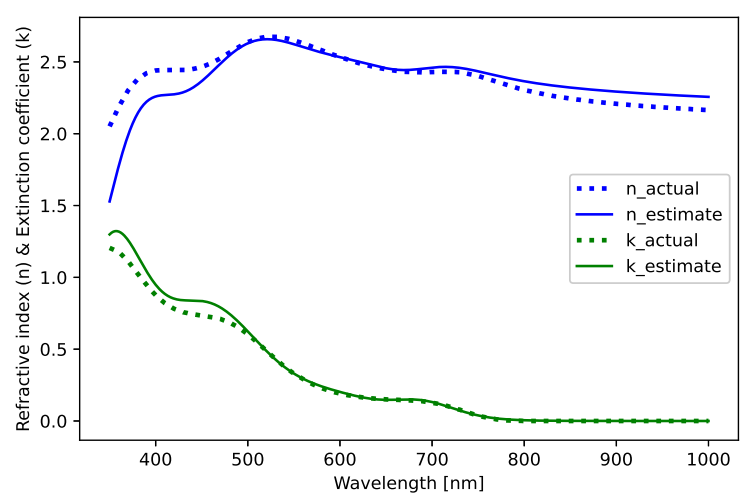

(d)

Figure 5. Inverse solutions obtained from optimized TLO and GO ensemble models applied on spectral data (synthetic) of specimen B: (a) Actual and estimated spectra from optimized TLO ensemble; total estimation loss is: 0.0335, (b) Actual and estimated refractive index and extinction coefficient extracted from optimized GO ensemble; actual and estimated thickness values are: $1350.0,1359.86 \mathrm{~nm}$, (c) Actual and estimated spectra from optimized GO ensemble; total estimation loss is: 0.0454, (d) Actual and estimated refractive index and extinction coefficient extracted from optimized GO ensemble; actual and estimated thickness values are: 169.0, $166.53 \mathrm{~nm}$. 


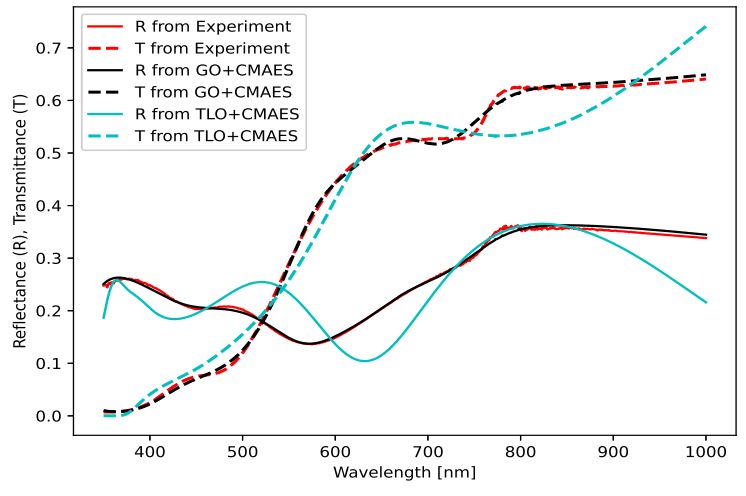

(a)

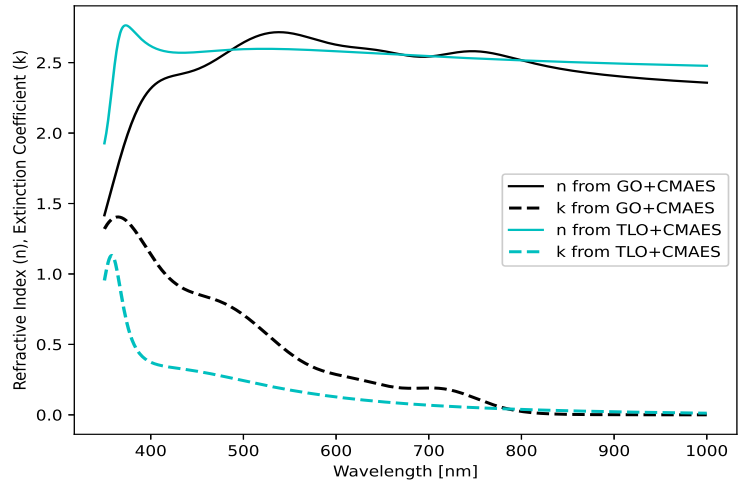

(b)

Figure 6. Estimated optical parameters from experimentally measured spectrophotometric data: (a) Measured vs. estimated spectra, (b) Refractive index and extinction coefficient estimates.

estimates simultaneously with $d$. The GO model can not be discarded based on its performance on the synthetic data in Table 2 because the same produces accurate estimates from the experimental data, as supported by Table 3 and Figure 6 . It is worth noting that a local search algorithm, such as sequential least square programming (SLSQP from 'scipy.optimize'), can be applied to the estimates found by an EA to further improve the solution accuracy if needed.

\section{Discussion on Results}

Inverse problems involve one-to-many mappings and often they are ill-posed ${ }^{35}$, therefore, finding exact solutions to such problems is challenging. Further, the difficulty level rises when inverse solutions are determined from noisy measurements. The present work demonstrates that the proposed optimization approach in combination with EAs does a reasonably good job in extracting accurate inverse solutions. The optical characteristics including the nature of complex refractive index profiles vary across diverse materials, such as inorganic, organic, glass and other miscellaneous materials. The number of oscillator components to be used in an ensemble model depends on the type of the concerned material. It is not trivial to come up with an universal rule of selecting the same; the current choice is subjective to two types of film materials: metal oxide and perovskite. In the present study, the number of oscillator components used to tackle type B thin-films are higher than that of type A thin-films because perovskite materials' refractive index profiles are more complex than metal oxides. In general, our solver application can be extended to a variety of materials by adjusting the number of composition elements in the optical models. The proposed evolutionary optimization approach does not require any prior learning or memory-based mapping. The successful runs take only about a few minutes to find a solution in an i7-4600M CPU@2.90 GHz processor. The present approach utilizes the spectral data at just one incident angle of light, however, in future, more spectral information (at multiple incident angles) can be added to alleviate the effect of uncertainty in experimental measurements. Also, there remains a scope of devising advanced techniques to tackle surface roughness and layer inhomogeneity.

\section{Conclusion}

The proposed optimization methodology using an evolutionary algorithm elegantly solves the inverse problem of determining optical parameters from spectrophotometric data. We achieve a quite satisfactory level of accuracy in estimating thin-films' thickness values. The employed evolutionary algorithm, CMAES, proves to be efficient in finding global solutions without spending much function evaluations. Interestingly, our proposed evolutionary optimization approach is able to extract the optical characteristics of the perovskite thin-films with both real and imaginary parts of the complex refractive index. In overall parameter estimation, the optimized Tauc-Lorentz ensemble performs well and the optimized Gaussian ensemble performs descent for metal oxide as well as perovskite thin-films. To this end, our proposed method can extract various thin-films' optical characteristics, i.e. thickness, real and imaginary refractive index, simultaneously from spectrophotometric data over a broad range of wavelengths, and it offers generality to be applicable for diverse materials. 


\section{Data Availability}

Data available upon reasonable request from Rajdeep Dutta (rajdeep_dutta@i2r.a-star.edu.sg) or Siyu Isaac Parker Tian (siyutian@u.nus.edu).

\section{References}

1. Loper, P. et al. Complex refractive index spectra of ch3nh3pbi3 perovskite thin films determined by spectroscopic ellipsometry and spectrophotometry. The journal physical chemistry letters 6, 66-71 (2014).

2. Nestler, P. \& Helm, C. Determination of refractive index and layer thickness of nm-thin films via ellipsometry. Opt. Express 25, 27077-27085 (2017).

3. Babeva, T., Kitova, S. \& Konstantinov, I. Photometric methods for determining the optical constants and the thicknesses of thin absorbing films: criteria for precise and unambiguous determination of $\mathrm{n}, \mathrm{k}$, and $\mathrm{d}$ in a wide spectral range. Appl. Opt. 40, 2682-2686 (2001).

4. Manley, P., Yin, G. \& Schmid, M. A method for calculating the complex refractive index of inhomogeneous thin films. $J$. Phys. D: Appl. Phys. 47, 205301 (2014).

5. Ruiz, R., Papadimitratos, A., Mayer, A. C. \& Malliaras, G. G. Thickness dependence of mobility in pentacene thin-film transistors. Adv. Mater. 17, 1795-1798 (2005).

6. Korotcenkov, G. \& Cho, B. K. Thin film sno2-based gas sensors: film thickness influence. Sensors Actuators B: Chem. 142, 321-330 (2009).

7. Xu, Z., Luo, Y., Liu, D., Shum, P. P. \& Sun, Q. Sensitivity-controllable refractive index sensor based on reflective -shaped microfiber resonator cooperated with vernier effect. Sci. reports 7, 1-8 (2017).

8. Nebioglu, A., Leon, J. A. \& Khudyakov, I. V. New uv-curable high refractive index oligomers. Ind. \& engineering chemistry research 47, 2155-2159 (2008).

9. Yepuri, V., Dubey, R. S. \& Kumar, B. Rapid and economic fabrication approach of dielectric reflectors for energy harvesting applications. Sci. Reports 10, 1-9 (2020).

10. Piegari, A. \& Masetti, E. Thin film thickness measurement: a comparison of various techniques. Thin solid films 124, 249-257 (1985).

11. Gao, L., Lemarchand, F. \& Lequime, M. Reverse engineering from spectrophotometric measurements: performances and efficiency of different optimization algorithms. Appl. Phys. A 108, 877-889 (2012).

12. Babeva, T., Kitova, S. \& Konstantinov, I. Photometric methods for determining the optical constants and the thicknesses of thin absorbing films: selection of a combination of photometric quantities on the basis of error analysis. Appl. Opt. 40, 2675-2681 (2001).

13. Katsidis, C. C. \& Siapkas, D. I. General transfer-matrix method for optical multilayer systems with coherent, partially coherent, and incoherent interference. Appl. Opt. 41, 3978-3987 (2002).

14. Lamprecht, K., Papousek, W. \& Leising, G. Problem of ambiguity in the determination of optical constants of thin absorbing films from spectroscopic reflectance and transmittance measurements. Appl. Opt. 36, 6364-6371 (1997).

15. Yin, G., Merschjann, C. \& Schmid, M. The effect of surface roughness on the determination of optical constants of cuinse and cugase thin films. J. Appl. Phys. 113, 213510 (2013).

16. Forouhi, A. \& Bloomer, I. Optical dispersion relations for amorphous semiconductors and amorphous dielectrics. Phys. review B 34, 7018 (1986).

17. Brimhall, N. et al. Measured optical constants of copper from $10 \mathrm{~nm}$ to $35 \mathrm{~nm}$. Opt. Express 17, 23873-23879 (2009).

18. Siyu Isaac, P. et al. Rapid and accurate thin film thickness extraction via uv-vis and machine learning. Proc. 47th IEEE Photovolt. Specialists Conf. (2020).

19. Kwak, H. et al. Non-destructive thickness characterisation of $3 \mathrm{~d}$ multilayer semiconductor devices using optical spectral measurements and machine learning. Light. Adv. Manuf. 2, 1-11 (2021).

20. Woollam, J. et al. Overview of variable-angle spectroscopic ellipsometry (vase): I. basic theory and typical applications. Opt. Metrol. A Critical Rev. 10294, 1029402 (1999).

21. Johs, B. et al. Overview of variable-angle spectroscopic ellipsometry (vase): Ii. advanced applications. Opt. Metrol. A Critical Rev. 10294, 1029404 (1999). 
22. Moré, J. J. The levenberg-marquardt algorithm: implementation and theory. Numer. analysis 105-116 (1978).

23. Hansen, N. The cma evolution strategy: A tutorial. arXiv preprint arXiv:1604.00772 (2016).

24. Fujii, G., Akimoto, Y. \& Takahashi, M. Exploring optimal topology of thermal cloaks by cma-es. Appl. Phys. Lett. 112, 061108 (2018).

25. Boggs, P. T. \& Tolle, J. W. Sequential quadratic programming. Acta numerica 4, 1-51 (1995).

26. Dorywalski, K., Schmidt-Gründ, R. \& Grundmann, M. Hybrid ga-gradient method for thin films ellipsometric data evaluation. J. Comput. Sci. 47, 101201 (2020).

27. Polgar, O., Fried, M., Lohner, T. \& Barsony, I. Comparison of algorithms used for evaluation of ellipsometric measurements random search, genetic algorithms, simulated annealing and hill climbing graph-searches. Surf. Sci. 457, 157-177 (2000).

28. Hansen, N. The cma evolution strategy: a comparing review. Towards a new evolutionary computation 75-102 (2006).

29. Jellison Jr, G. E. \& Modine, F. A. Parameterization of the optical functions of amorphous materials in the interband region. Appl. Phys. Lett. 69, 371-373 (1996).

30. Forouhi, A. R. \& Bloomer, I. Optical properties of crystalline semiconductors and dielectrics. Phys. review B 38, 1865-1874 (1988).

31. Ohta, K. \& Ishida, H. Comparison among several numerical integration methods for kramers-kronig transformation. Appl. Spectrosc. 42, 952-957 (1988).

32. Canit, J. C., Billardon, M. \& Badoz, J. P. Relationship between the real and imaginary parts of the refractive index. JOSA 59, 1000-1001 (1969).

33. Loper, P. et al. Complex refractive index spectra of $\mathrm{CH}_{3} \mathrm{NH}_{3} \mathrm{PbI}_{3}$ perovskite thin films determined by spectroscopic ellipsometry and spectrophotometry. The journal physical chemistry letters 6, 66-71 (2015).

34. MacLeod, B. P. et al. Self-driving laboratory for accelerated discovery of thin-film materials. Sci. Adv. 6, eaaz8867 (2020).

35. O'Sullivan, F. A statistical perspective on ill-posed inverse problems. Stat. Sci. 1, 502-518 (1986).

\section{Acknowledgement}

This study is supported by the Accelerated Materials Development for Manufacturing Program at A*STAR via the AME Programmatic Fund by the Agency for Science, Technology and Research under Grant No. A1898b0043, (R.D., S.J.) and National Research Foundation, Prime Ministers Office, Singapore under its Campus for Research Excellence and Technological Enterprise (CREATE) program through the Singapore Massachusetts Institute of Technology (MIT) Alliance for Research and Technologys Low Energy Electronic Systems research program (S.T., Z.L., T.B.).

\section{Author Contributions}

R.D., and S.T. contributed equally for this work; R.D., S.T., T.B., and S.J. conceived the inverse problem; R.D. developed and validated optimization algorithms with key intellectual contributions from S.T., Z.L., T.B., and S.J.; S.T., Z.L., T.B. prepared the simulation-based synthetic data and S.V., Y.C., D.B. conducted the experiments; R.D. wrote the manuscript with suggestions from all the co-authors; T.B. and S.J. supervised the underlying research.

\section{Additional Information}

\section{Competing Interests}

The authors declare no competing interests. 